\title{
Partial Coupled Fixed Points and Coupled Fixed Points
}

\author{
Marta Demma ${ }^{1}$, Peyman Salimi ${ }^{2}$ and Pasquale Vetro ${ }^{{ }^{*}}$ \\ ${ }^{1}$ Dipartimento di Matematica e Informatica, University of Palermo, Via Archirafi n. 34, 90123 Palermo (Italy) \\ ${ }^{2}$ Department of Mathematics, Sahand University of Technology, Tabriz (Iran) \\ Email: pasquale.vetro@unipa.it
}

\begin{abstract}
We establish partial coupled fixed point and coupled fixed point results for mixed monotone mappings satisfying a generalized contractive condition in ordered partial metric spaces and ordered metric spaces. Our results generalize some interesting coupled fixed point theorems obtained in [T. G. Bhaskar and V. Lakshmikantham, "Fixed point theorems in partially ordered metric spaces and applications", Nonlinear Anal., vol. 65, pp. 1379-1393, 2006].
\end{abstract}

Keywords: Partial coupled fixed point, coupled fixed point, mixed monotone property, partially ordered set, ordered partial metric spaces, ordered metric spaces.

\section{Notation}

$\begin{array}{ll}\text { Section } 3 & \\ \preceq, \leq & \text { order relations; } \\ X & \text { non-empty set; } \\ x, y, z, x_{1}, y_{1}, x_{2}, y_{2} & \text { elements of } X ; \\ x_{n}, x_{m} & \text { elements of sequence in } X ; \\ p, p^{s}, d & \text { functions on } X \times X ; \\ \tau_{p} & \text { topology induced by } p ; \\ \varepsilon & \text { positive real number; } \\ B_{p}(x, \varepsilon) & \text { open ball on } X ; \\ T & \text { self-mapping on } X ; \\ F & \text { mapping on } X \times X \text { to } X . \\ & \\ \text { Section } 4 & \\ \text { inf } & \text { infimum; } \\ \Gamma & \text { functional term; } \\ m, n, n_{0} & \text { indices; } \\ S, P_{0}, P_{n}, P_{0,1}, P_{m, n}, \text { etc } & \text { symbolic assignments for constructive procedure; } \\ \left(x^{*}, y^{*}\right),\left(u^{*}, v^{*}\right) & \text { coupled fixed points; } \\ \lim \sup & \text { superior limit operator. } \\ \text { Section } 5 & \\ 5 & \\ D & \text { order relation; } \\ H & \text { function on } X^{2} \times X^{2} ; \\ & \text { technical function; } \\ & \text { self-mapping on } X^{2} . \\ & \end{array}$

\section{Introduction}

In the last years, fixed points of mappings in partially ordered metric spaces have been investigated by many researchers $[1,2,3,5,6,8,9,10,12,13,14,16,17,21,23,24,26,27]$. The first result in this direction was given by Ran and Reurings [20, Theorem 2.1] who presented its applications to linear and nonlinear metric spaces. Subsequently, Nieto and Rodríguez-López [16] extended the result of Ran and Reurings [20] for non-decreasing mappings and applied it to obtain a unique solution for a first order ordinary differential 
equation with periodic boundary conditions. Similar applications based on a version of Theorems 2.1-2.5 [16] for a mixed monotone mapping $F: X \times X \rightarrow X$ were given by Bhaskar and Lakshmikantham [4]. In [4], the authors introduced the notion of a coupled fixed point and proved some coupled fixed point theorems for mappings satisfying a mixed monotone property. They discussed the problem of uniqueness of coupled fixed point and applied their theorems to problems of existence and uniqueness of solution for a periodic boundary value problem.

In recent years, in the setting of domain theory, attempts were made in order to equip semantics domain with a notion of distance. In particular, Matthews [11] introduced the notion of a partial metric space as a part of the study of denotational semantics of data for networks, showing that the contraction mapping principle of Banach can be generalized to the partial metric context for applications in program verification. Moreover, the existence of several connections between partial metrics and topological aspects of domain theory has been lately pointed by some authors as O'Neill [15] and others. Subsequently, several authors $[7,18,19,22,25]$ have proved fixed point theorems in partial metric spaces.

In this paper, we generalize the results of Bhaskar and Lakshmikantham [4] by considering generalized contractive conditions for mixed monotone mappings, in the framework of ordered partial metric spaces and ordered metric spaces, and prove results concerning partial coupled fixed point and coupled fixed point. We give also an example to illustrate our results.

\section{Preliminaries}

Let $(X, \preceq)$ be a partially ordered set and $T: X \rightarrow X$ be a mapping. The mapping $T$ is said to be non-decreasing if for all $x, y \in X, x \preceq y$ implies $T(x) \preceq T(y)$. Similarly, $T$ is said to be non-increasing, if for all $x, y \in X, x \preceq y$ implies $T(x) \succeq T(y)$.

Now, we recall the notions of mixed monotone mapping and coupled fixed point introduced by Bhaskar and Lakshmikantham [4].

Definition 1. Let $(X, \preceq)$ be a partially ordered set and $F: X \times X \rightarrow X$. The mapping $F$ is said to have the mixed monotone property if $F$ is monotone non-decreasing in its first argument and is monotone non-increasing in its second argument, that is, for all $x_{1}, x_{2} \in X, x_{1} \preceq x_{2}$ implies $F\left(x_{1}, y\right) \preceq F\left(x_{2}, y\right)$, for any $y \in X$ and for all $y_{1}, y_{2} \in X, y_{1} \preceq y_{2}$ implies $F\left(x, y_{1}\right) \succeq F\left(x, y_{2}\right)$, for any $x \in X$.

Definition 2. An element $(x, y) \in X \times X$ is called a coupled fixed point of the mapping $F: X \times X \rightarrow X$ if $F(x, y)=x$ and $F(y, x)=y$.

Now, we recall some definitions and some properties of a partial metric space $[7,11,15,18,22,25]$. A partial metric on a nonempty set $X$ is a function $p: X \times X \rightarrow[0,+\infty)$ such that for all $x, y, z \in X$ :

$\left(p_{1}\right) x=y \Leftrightarrow p(x, x)=p(x, y)=p(y, y)$,

$\left(p_{2}\right) p(x, x) \leq p(x, y)$,

$\left(p_{3}\right) p(x, y)=p(y, x)$,

$\left(p_{4}\right) p(x, y) \leq p(x, z)+p(z, y)-p(z, z)$.

A partial metric space is a pair $(X, p)$ such that $X$ is a nonempty set and $p$ is a partial metric on $X$. It is clear that, if $p(x, y)=0$, then from $\left(p_{1}\right)$ and $\left(p_{2}\right)$ it follows that $x=y$. But if $x=y, p(x, y)$ may not be 0 . A basic example of a partial metric space is the pair $([0,+\infty), p)$, where $p(x, y)=\max \{x, y\}$ for all $x, y \in[0,+\infty)$. Other examples of partial metric spaces which are interesting from a computational point of view can be found in [11].

Each partial metric $p$ on $X$ generates a $T_{0}$ topology $\tau_{p}$ on $X$ which has as a base the family of open $p$-balls $\left\{B_{p}(x, \varepsilon): x \in X, \varepsilon>0\right\}$, where

$$
B_{p}(x, \varepsilon)=\{y \in X: p(x, y)<p(x, x)+\varepsilon\},
$$

for all $x \in X$ and $\varepsilon>0$.

If $p$ is a partial metric on $X$, then the function $p^{s}: X \times X \rightarrow[0,+\infty)$ given by

$$
p^{s}(x, y)=2 p(x, y)-p(x, x)-p(y, y)
$$


is a metric on $X$. Let $(X, p)$ be a partial metric space. Then:

A sequence $\left\{x_{n}\right\}$ in a partial metric space $(X, p)$ converges to a point $x \in X$ if and only if $p(x, x)=$ $\lim _{n \rightarrow+\infty} p\left(x, x_{n}\right)$.

A sequence $\left\{x_{n}\right\}$ in a partial metric space $(X, p)$ is called a Cauchy sequence if there exists (and is finite) $\lim _{n, m \rightarrow+\infty} p\left(x_{n}, x_{m}\right)$.

A partial metric space $(X, p)$ is said to be complete if every Cauchy sequence $\left\{x_{n}\right\}$ in $X$ converges, with respect to $\tau_{p}$, to a point $x \in X$ such that $p(x, x)=\lim _{n, m \rightarrow+\infty} p\left(x_{n}, x_{m}\right)$.

A sequence $\left\{x_{n}\right\}$ in a partial metric space $(X, p)$ is called 0-Cauchy if $\lim _{n \rightarrow+\infty} p\left(x_{n}, x_{m}\right)=0$. We say that $(X, p)$ is 0-complete if every 0-Cauchy sequence in $X$ converges, with respect to $\tau_{p}$, to a point $x \in X$ such that $p(x, x)=0$.

On the other hand, the partial metric space $(\mathbb{Q} \cap[0,+\infty), p)$, where $\mathbb{Q}$ denotes the set of rational numbers and the partial metric $p$ is given by $p(x, y)=\max \{x, y\}$, provides an example of a 0 -complete partial metric space which is not complete.

It is easy to see that, every closed subset of a complete (0-complete) partial metric space is complete (0-complete).

Lemma 3 ([11,18]). Let $(X, p)$ be a partial metric space.

(a) $\left\{x_{n}\right\}$ is a Cauchy sequence in $(X, p)$ if and only if it is a Cauchy sequence in the metric space $\left(X, p^{s}\right)$.

(b) A partial metric space $(X, p)$ is complete if and only if the metric space $\left(X, p^{s}\right)$ is complete. Furthermore, $\lim _{n \rightarrow+\infty} p^{s}\left(x_{n}, x\right)=0$ if and only if

$$
p(x, x)=\lim _{n \rightarrow+\infty} p\left(x_{n}, x\right)=\lim _{n, m \rightarrow+\infty} p\left(x_{n}, x_{m}\right) .
$$

Definition 4. Let $(X, p)$ be a partial metric space and $F: X \times X \rightarrow X$ be a function. An element $(x, y)$ is called a partial coupled fixed point of $F$ if

$$
p(x, F(x, y))+p(y, F(y, x))=p(x, x)+p(y, y) .
$$

Note that, if $(X, p)$ is a metric space then $(x, y)$ is a usual coupled fixed point of $F$.

Example 5. Let $X=[0,1], p(x, y)=|x-y|$ if $x, y \in[0,1)$ and $p(1, y)=1$ for all $y \in X$, and $F: X \times X \rightarrow X$ be defined by

$$
F(x, y)= \begin{cases}\frac{x-y}{2} & \text { if } x \geq y \\ 0 & \text { if } x<y\end{cases}
$$

The mapping $F$ has the following partial coupled fixed points:

$$
(0,0), \quad(0,1), \quad(1,0), \quad(1,1) .
$$

Note that only $(0,0)$ is a coupled fixed point for $F$.

Definition 6. Let $X$ be a nonempty set. Then $(X, p, \preceq)$ is called an ordered partial metric space if $(X, \preceq)$ is a partially ordered set, and $(X, p)$ is a partial metric space. If $p=d$ is a metric, then $(X, d, \preceq)$ is an ordered metric space.

\section{Main Results}

The first main result in this paper is the following partial coupled result which generalizes Theorem 2.2 in $[4]$.

Theorem 7. Let $(X, p, \preceq)$ be a complete ordered partial metric space and $F: X \times X \rightarrow X$ be a mixed monotone mapping. Assume that there exists $r \in[0,1)$ such that

$$
p(F(x, y), F(u, v)) \leq \frac{1}{2} \max \{r[p(x, u)+p(y, v)], p(x, x)+p(y, y), p(u, u)+p(v, v)\}
$$

for all $x, y, u, v \in X$ with $u \succeq x$ and $y \succeq v$. If the following conditions hold: 
(i) if a non-decreasing sequence $\left\{x_{n}\right\}$ is such that $x_{n} \rightarrow x$, then $x_{n} \preceq x$ for all $n \in \mathbb{N}$,

(ii) if a non-increasing sequence $\left\{y_{n}\right\}$ is such that $y_{n} \rightarrow y$, then $y_{n} \succeq y$ for all $n \in \mathbb{N}$,

(iii) there exist $x_{0}, y_{0} \in X$ such that $x_{0} \preceq F\left(x_{0}, y_{0}\right)$ and $y_{0} \succeq F\left(y_{0}, x_{0}\right)$.

Then, $F$ has a partial coupled fixed point $\left(x^{*}, y^{*}\right)$. Moreover, if $p\left(x^{*}, x^{*}\right)=p\left(y^{*}, y^{*}\right)=0$, then $\left(x^{*}, y^{*}\right)$ is a coupled fixed point for $F$.

Proof. Let $x_{0}, y_{0} \in X$ such that $x_{0} \preceq F\left(x_{0}, y_{0}\right)$ and $y_{0} \succeq F\left(y_{0}, x_{0}\right)$. Define $x_{1}, y_{1} \in X$ such that $x_{1}=F\left(x_{0}, y_{0}\right)$ and $y_{1}=F\left(y_{0}, x_{0}\right)$. Similarly $x_{2}=F\left(x_{1}, y_{1}\right)$ and $y_{2}=F\left(y_{1}, x_{1}\right)$. We construct two sequences $\left\{x_{n}\right\}$ and $\left\{y_{n}\right\}$ such that,

$$
x_{n+1}=F\left(x_{n}, y_{n}\right), \quad y_{n+1}=F\left(y_{n}, x_{n}\right) \quad \text { for all } \quad n \geq 0 .
$$

Let us prove that

$$
x_{n} \preceq x_{n+1} \text { and } y_{n} \succeq y_{n+1} \text { for all } n \geq 0 \text {. }
$$

Since

$$
x_{0} \preceq F\left(x_{0}, y_{0}\right)=x_{1} \text { and } y_{0} \succeq F\left(y_{0}, x_{0}\right)=y_{1},
$$

then (2) holds for $n=0$. Suppose that (2) holds for some $n \geq 1$. Since $F$ has the mixed monotone property, then

$$
x_{n+1}=F\left(x_{n}, y_{n}\right) \preceq F\left(x_{n+1}, y_{n}\right)
$$

and

$$
F\left(y_{n+1}, x_{n}\right) \preceq F\left(y_{n}, x_{n}\right)=y_{n+1} .
$$

Also for the same reason we have

$$
x_{n+2}=F\left(x_{n+1}, y_{n+1}\right) \succeq F\left(x_{n+1}, y_{n}\right)
$$

and

$$
F\left(y_{n+1}, x_{n}\right) \succeq F\left(y_{n+1}, x_{n+1}\right)=y_{n+2} .
$$

Observe that from the previous inequalities we can derive $x_{n+1} \preceq x_{n+2}$ and $y_{n+1} \succeq y_{n+2}$. By the principle of mathematical induction it follows that (2) holds for all $n \geq 0$.

Now, from (1) with $x=u$ and $y=v$ we deduce

$$
p(F(x, y), F(x, y)) \leq \frac{1}{2}[p(x, x)+p(y, y)] \text { for all } \quad x, y \in X .
$$

Since (3) holds for all $x, y \in X$, then

$$
p(F(y, x), F(y, x)) \leq \frac{1}{2}[p(x, x)+p(y, y)] \text { for all } \quad x, y \in X
$$

and hence by (3) and (4), we get

$$
p(F(x, y), F(x, y))+p(F(y, x), F(y, x)) \leq p(x, x)+p(y, y) \quad \text { for all } \quad x, y \in X .
$$

By taking $x=x_{n+1}$ and $y=y_{n+1}$ in (5), we obtain

$$
\begin{aligned}
p\left(x_{n+1}, x_{n+1}\right)+p\left(y_{n+1}, y_{n+1}\right) & =p\left(F\left(x_{n}, y_{n}\right), F\left(x_{n}, y_{n}\right)\right)+p\left(F\left(y_{n}, x_{n}\right), F\left(y_{n}, x_{n}\right)\right) \\
& \leq p\left(x_{n}, x_{n}\right)+p\left(y_{n}, y_{n}\right),
\end{aligned}
$$

for all $n \geq 0$. Thus $\left\{p\left(x_{n}, x_{n}\right)+p\left(y_{n}, y_{n}\right)\right\}_{n \geq 0}$ is a non-increasing sequence. Put

$$
\begin{gathered}
P_{n}=p\left(x_{n}, x_{n}\right)+p\left(y_{n}, y_{n}\right) \quad \text { and } \quad P_{n, m}=p\left(x_{n}, x_{m}\right)+p\left(y_{n}, y_{m}\right) \quad \text { for all } n, m \in \mathbb{N}, \\
S:=\lim _{n \rightarrow+\infty} P_{n}=\inf _{n \in \mathbb{N}} P_{n}
\end{gathered}
$$


and

$$
\Gamma=P_{0}+\frac{1}{1-r} P_{0,1}
$$

Let us prove that

$$
P_{m, n} \leq 2 \Gamma \text { for given } m, n \in \mathbb{N} \text {. }
$$

At first

$$
\begin{aligned}
p\left(x_{0}, x_{n}\right) & \leq p\left(x_{0}, x_{1}\right)+p\left(F\left(x_{0}, y_{0}\right), F\left(x_{n-1}, y_{n-1}\right)\right) \\
& \leq p\left(x_{0}, x_{1}\right)+\frac{1}{2} \max \left\{r P_{0, n-1}, P_{0}, P_{n-1}\right\} .
\end{aligned}
$$

Similarly

$$
\begin{aligned}
p\left(y_{0}, y_{n}\right) & \leq p\left(y_{0}, y_{1}\right)+p\left(F\left(y_{0}, x_{0}\right), F\left(y_{n-1}, x_{n-1}\right)\right) \\
& \leq p\left(y_{0}, y_{1}\right)+\frac{1}{2} \max \left\{r P_{0, n-1}, P_{0}, P_{n-1}\right\}
\end{aligned}
$$

and so

$$
\begin{aligned}
P_{0, n} & \leq P_{0,1}+\max \left\{r P_{0, n-1}, P_{0}, P_{n-1}\right\} \\
& \leq P_{0,1}+\max \left\{r P_{0, n-1}, P_{0}, P_{n-2}\right\} \\
& \leq P_{0,1}+\max \left\{r P_{0, n-1}, r P_{0,1}+P_{0}, r P_{0,1}+P_{n-2}\right\} .
\end{aligned}
$$

Since,

$$
P_{0, n-1} \leq P_{0,1}+\max \left\{r P_{0, n-2}, P_{0}, P_{n-2}\right\},
$$

from the previous inequality, we get

$$
P_{0, n} \leq P_{0,1}+r P_{0,1}+\max \left\{r^{2} P_{0, n-2}, P_{0}, P_{n-2}\right\} .
$$

By continuing this process, we obtain

$$
\begin{aligned}
P_{0, n} & \leq\left(1+r+\cdots+r^{n-1}\right) P_{0,1}+P_{0} \\
& \leq \frac{r}{1-r} P_{0,1}+P_{0}=\Gamma .
\end{aligned}
$$

Consequently

$$
P_{m, n} \leq P_{0, m}+P_{0, n} \leq 2 \Gamma \quad \text { for all } m, n \in \mathbb{N}
$$

and (6) holds.

Given any $\epsilon>0$, from the definition of $S$ and $0 \leq r<1$, there exists $n_{0} \in \mathbb{N}$ such that $P_{n_{0}}, 2 \Gamma r^{n_{0}}<S+\epsilon$. Therefore, for all $m, n \geq 2 n_{0}$

$$
\begin{aligned}
p\left(x_{m}, x_{n}\right) & =p\left(F\left(x_{m-1}, y_{m-1}\right), F\left(x_{n-1}, y_{n-1}\right)\right) \\
& \leq \frac{1}{2} \max \left\{r P_{m-1, n-1}, P_{m-1}, P_{n-1}\right\} .
\end{aligned}
$$

Similarly

$$
p\left(y_{m}, y_{n}\right) \leq \frac{1}{2} \max \left\{r P_{m-1, n-1}, P_{m-1}, P_{n-1}\right\}
$$

Then, we get

$$
\begin{aligned}
P_{m, n} & \leq \max \left\{r P_{m-1, n-1}, P_{m-1}, P_{n-1}\right\} \\
& \leq \max \left\{r P_{m-1, n-1}, P_{m-2}, P_{n-2}\right\} .
\end{aligned}
$$


As

$$
P_{m-1, n-1} \leq \max \left\{r P_{m-2, n-2}, P_{m-2}, P_{n-2}\right\},
$$

by $(7)$, we deduce that

$$
P_{m, n} \leq \max \left\{r^{2} P_{m-2, n-2}, P_{m-2}, P_{n-2}\right\} .
$$

By continuing this process, we get

$$
\begin{aligned}
P_{m, n} & \leq \max \left\{r^{n_{0}} P_{m-n_{0}, n-n_{0}}, P_{m-n_{0}}, P_{n-n_{0}}\right\} \\
& <S+\epsilon .
\end{aligned}
$$

Then, $S=\lim _{m, n \rightarrow+\infty} P_{m, n}$. From

$$
\begin{aligned}
\lim _{m \rightarrow n \rightarrow+\infty}\left[p^{s}\left(x_{m}, x_{n}\right)+p^{s}\left(y_{m}, y_{n}\right)\right] & =\lim _{m, n \rightarrow+\infty}\left[2 P_{m, n}-P_{m}-P_{n}\right] \\
& =2 S-S-S=0,
\end{aligned}
$$

we deduce that $\left\{x_{n}\right\}$ and $\left\{y_{n}\right\}$ are two Cauchy sequences in $\left(X, p^{s}\right)$ and hence in $(X, p)$. Since $(X, p)$ is complete, then there exist $x^{*}, y^{*} \in X$ such that $x_{n} \rightarrow x^{*}$ and $y_{n} \rightarrow y^{*}$, that is,

$$
p\left(x^{*}, x^{*}\right)=\lim _{n \rightarrow+\infty} p\left(x^{*}, x_{n}\right)=\lim _{n \rightarrow+\infty} p\left(x_{n}, x_{m}\right),
$$

and

$$
p\left(y^{*}, y^{*}\right)=\lim _{n \rightarrow+\infty} p\left(y^{*}, y_{n}\right)=\lim _{n \rightarrow+\infty} p\left(y_{n}, y_{m}\right) .
$$

Also from $(i)$ and $(i i)$, we have $x_{n} \preceq x^{*}$ and $y_{n} \succeq y^{*}$. Hence

$$
p\left(x^{*}, F\left(x^{*}, y^{*}\right)\right) \leq p\left(x^{*}, x_{n}\right)+p\left(x_{n}, F\left(x^{*}, y^{*}\right)\right)-p\left(x_{n}, x_{n}\right) .
$$

By taking the limit as $n \rightarrow+\infty$ in the above inequality, we get

$$
p\left(x^{*}, F\left(x^{*}, y^{*}\right)\right) \leq \liminf _{n \rightarrow+\infty} p\left(x_{n}, F\left(x^{*}, y^{*}\right)\right)
$$

Similarly

$$
p\left(y^{*}, F\left(y^{*}, x^{*}\right)\right) \leq \liminf _{n \rightarrow+\infty} p\left(y_{n}, F\left(y^{*}, x^{*}\right)\right)
$$

On the other hand, from (1), we have

$$
\begin{aligned}
& \limsup _{n \rightarrow+\infty} p\left(x_{n}, F\left(x^{*}, y^{*}\right)\right) \\
= & \limsup _{n \rightarrow+\infty} p\left(F\left(x_{n-1}, y_{n-1}\right), F\left(x^{*}, y^{*}\right)\right) \\
\leq & \limsup _{n \rightarrow+\infty} \frac{1}{2} \max \left\{r\left[p\left(x_{n-1}, x^{*}\right)+p\left(y_{n-1}, y^{*}\right)\right], P_{n-1}, p\left(x^{*}, x^{*}\right)+p\left(y^{*}, y^{*}\right)\right\} \\
= & \frac{1}{2}\left[p\left(x^{*}, x^{*}\right)+p\left(y^{*}, y^{*}\right)\right] .
\end{aligned}
$$

Similarly

$$
\limsup _{n \rightarrow+\infty} p\left(y_{n}, F\left(y^{*}, x^{*}\right)\right) \leq \frac{1}{2}\left[p\left(x^{*}, x^{*}\right)+p\left(y^{*}, y^{*}\right)\right]
$$

Then, we have

$$
p\left(x^{*}, F\left(x^{*}, y^{*}\right)\right)+p\left(y^{*}, F\left(y^{*}, x^{*}\right)\right) \leq p\left(x^{*}, x^{*}\right)+p\left(y^{*}, y^{*}\right),
$$

and since

we obtain

$$
p\left(x^{*}, x^{*}\right)+p\left(y^{*}, y^{*}\right) \leq p\left(x^{*}, F\left(x^{*}, y^{*}\right)\right)+p\left(y^{*}, F\left(y^{*}, x^{*}\right)\right),
$$

$$
p\left(x^{*}, F\left(x^{*}, y^{*}\right)\right)+p\left(y^{*}, F\left(y^{*}, x^{*}\right)\right)=p\left(x^{*}, x^{*}\right)+p\left(y^{*}, y^{*}\right) .
$$

Hence $\left(x^{*}, y^{*}\right)$ is a partial coupled fixed point of $F$. If $p\left(x^{*}, x^{*}\right)=p\left(y^{*}, y^{*}\right)=0$, then $p\left(x^{*}, F\left(x^{*}, y^{*}\right)\right)=$ $p\left(y^{*}, F\left(y^{*}, x^{*}\right)\right)=0$ and thus $x^{*}=F\left(x^{*}, y^{*}\right)$ and $y^{*}=F\left(y^{*}, x^{*}\right)$, that is, $\left(x^{*}, y^{*}\right)$ is a coupled fixed point for $F$. 
From Theorem 7, we deduce the following theorem.

Theorem 8. Let $(X, p, \preceq)$ be a complete ordered partial metric space and $F: X \times X \rightarrow X$ be a mixed monotone mapping. Assume that there exists $r \in[0,1)$ such that

$$
p(F(x, y), F(u, v)) \leq \frac{1}{2} \max \left\{r[p(x, u)+p(y, v)], \frac{p(x, x)+p(y, y)+p(u, u)+p(v, v)}{2}\right\}
$$

for all $x, y, u, v \in X$ with $u \succeq x$ and $y \succeq v$. If the following conditions hold:

(i) if a non-decreasing sequence $\left\{x_{n}\right\}$ is such that $x_{n} \rightarrow x$, then $x_{n} \preceq x$ for all $n \in \mathbb{N}$,

(ii) if a non-increasing sequence $\left\{y_{n}\right\}$ is such that $y_{n} \rightarrow y$, then $y_{n} \succeq y$ for all $n \in \mathbb{N}$,

(iii) there exist $x_{0}, y_{0} \in X$ such that $x_{0} \preceq F\left(x_{0}, y_{0}\right)$ and $y_{0} \succeq F\left(y_{0}, x_{0}\right)$,

then $F$ has a partial coupled fixed point $\left(x^{*}, y^{*}\right)$. Moreover, if $p\left(x^{*}, x^{*}\right)=p\left(y^{*}, y^{*}\right)=0$, then $\left(x^{*}, y^{*}\right)$ is a coupled fixed point for $F$.

Proof. Since

$$
\frac{p(x, x)+p(y, y)+p(u, u)+p(v, v)}{2} \leq \max \{p(x, x)+p(y, y), p(u, u)+p(v, v)\},
$$

by (8), we have

$$
p(F(x, y), F(u, v)) \leq \frac{1}{2} \max \{r[p(x, u)+p(y, v)], p(x, x)+p(y, y), p(u, u)+p(v, v)\},
$$

for all $x, y, u, v \in X$ with $u \succeq x$ and $y \succeq v$. Thus the hypotheses of Theorem 7 are satisfied and therefore $F$ has a partial coupled fixed point $\left(x^{*}, y^{*}\right)$, which is a coupled fixed point if $p\left(x^{*}, x^{*}\right)=p\left(y^{*}, y^{*}\right)=0$.

Corollary 9 (Theorem 2.2 of $[4])$. Let $(X, d, \preceq)$ be a complete ordered metric space and $F: X \times X \rightarrow X$ be a mixed monotone mapping. Assume that there exists $r \in[0,1)$ such that

$$
d(F(x, y), F(u, v)) \leq \frac{r}{2}[d(x, u)+d(y, v)]
$$

for all $x, y, u, v \in X$ with $u \succeq x$ and $y \succeq v$. If the following conditions hold:

(i) if a non-decreasing sequence $\left\{x_{n}\right\}$ is such that $x_{n} \rightarrow x$, then $x_{n} \preceq x$ for all $n \in \mathbb{N}$;

(ii) if a non-increasing sequence $\left\{y_{n}\right\}$ is such that $y_{n} \rightarrow y$, then $y_{n} \succeq y$ for all $n \in \mathbb{N}$;

(iii) there exist $x_{0}, y_{0} \in X$ such that $x_{0} \preceq F\left(x_{0}, y_{0}\right)$ and $y_{0} \succeq F\left(y_{0}, x_{0}\right)$;

Then $F$ has a coupled fixed point.

Example 10. Let $X=[0,2], d(x, y)=|x-y|, p(x, y)=\max \{x, y\}$ and $F: X \times X \rightarrow X$ be defined by

$$
F(x, y)= \begin{cases}\frac{x-y}{2} & \text { if } x \geq y \\ 0 & \text { if } x<y\end{cases}
$$

Then $F$ is mixed monotone and satisfies condition (1) but does not satisfy condition (9). Indeed, assume there exists $r \in[0,1)$, such that (9) holds. This means

$$
\left|\frac{x-y}{2}-\frac{u-v}{2}\right| \leq \frac{r}{2}|x-u|+\frac{r}{2}|y-v|,
$$

for all $x, y, u, v \in X$ with $u \geq x \geq y \geq v$, by which, for $x=u$, we get

$$
\frac{1}{2}|y-v| \leq \frac{r}{2}|y-v|,
$$

which for $y>v$ would imply $1 \leq r$, a contradiction.

Now we prove that (1) holds. Consider the following cases: 
(1) $u \geq x \geq y \geq v$

(2) $u \geq x, y \geq v$ with $x<y$ and $u \geq v$,

(3) $u \geq x, y \geq v$ with $x<y$ and $u<v$.

Case 1. Under this assumption, we get that

$$
\begin{aligned}
p(F(x, y), F(u, v)) & =p\left(\frac{x-y}{2}, \frac{u-v}{2}\right) \\
& =\frac{u-v}{2} \leq \frac{u+v}{2} \\
& =\frac{1}{2}[p(u, u)+p(v, v)],
\end{aligned}
$$

and hence (1) holds.

Case 2. In this case

$$
\begin{aligned}
p(F(x, y), F(u, v)) & =p\left(0, \frac{u-v}{2}\right) \\
& =\frac{u-v}{2} \leq \frac{1}{2}[p(u, u)+p(v, v)],
\end{aligned}
$$

and hence (1) holds.

Case 3. In this case $p(F(x, y), F(u, v))=0$ and hence (1) holds.

By Theorem 7 , we obtain that $F$ has a partial coupled fixed point $(0,0)$ that is a coupled fixed point. Theorem 2.2 of [4] cannot be applied to $F$ in this example.

Theorem 11. Let $(X, p, \preceq)$ be a complete ordered partial metric space and $F: X \times X \rightarrow X$ be a mixed monotone mapping. Assume that there exists $r \in[0,1)$ such that

$$
p(F(x, y), F(u, v)) \leq \frac{1}{2} \max \{r[p(x, u)+p(y, v)], p(x, x)+p(y, y), p(u, u)+p(v, v)\}
$$

for all $x, y, u, v \in X$ with $u \succeq x$ and $y \succeq v$. If the following conditions hold:

(i) $F$ is continuous with respect to $\tau_{p}$,

(ii) there exist $x_{0}, y_{0} \in X$ such that $x_{0} \preceq F\left(x_{0}, y_{0}\right)$ and $y_{0} \succeq F\left(y_{0}, x_{0}\right)$,

then $F$ has a coupled fixed point.

Proof. Proceeding exactly as in Theorem 7 , we have that $\left\{x_{n}\right\}$ and $\left\{y_{n}\right\}$ are Cauchy sequences in the complete partial metric space $(X, p)$. Then there exist $x^{*}, y^{*} \in X$ such that $x_{n} \rightarrow x^{*}$ and $y_{n} \rightarrow y^{*}$. Since $F$ is continuous, then we have

$$
x^{*}=\lim _{n \rightarrow+\infty} x_{n}=\lim _{n \rightarrow+\infty} F\left(x_{n-1}, y_{n-1}\right)=F\left(x^{*}, y^{*}\right) .
$$

Similarly

$$
y^{*}=F\left(y^{*}, x^{*}\right) .
$$

That is, $\left(x^{*}, y^{*}\right)$ is a coupled fixed point of $F$.

Similarly, one can obtain the following theorem.

Theorem 12. Let $(X, p, \preceq)$ be a complete ordered partial metric space and $F: X \times X \rightarrow X$ be a mixed monotone mapping. Assume that there exists $r \in[0,1)$ such that

$$
p(F(x, y), F(u, v)) \leq \frac{1}{2} \max \left\{r[p(x, u)+p(y, v)], \frac{p(x, x)+p(y, y)+p(u, u)+p(v, v)}{2}\right\}
$$

for all $x, y, u, v \in X$ with $u \succeq x$ and $y \succeq v$. If the following conditions hold:

(i) $F$ is continuous with respect to $\tau_{p}$, 
(ii) there exist $x_{0}, y_{0} \in X$ such that $x_{0} \preceq F\left(x_{0}, y_{0}\right)$ and $y_{0} \succeq F\left(y_{0}, x_{0}\right)$.

Then, $F$ has a coupled fixed point. Moreover, if $\left(x^{*}, y^{*}\right)$ and $\left(u^{*}, v^{*}\right)$ are two coupled fixed points for $F$, then $x^{*}=u^{*}$ and $y^{*}=v^{*}$ if and only if $x^{*}$ is comparable with $u^{*}$ and $y^{*}$ is comparable with $v^{*}$.

Proof. Proceeding exactly as in Theorem 8, we deduce that the hypotheses of Theorem 11 are satisfied and therefore $F$ has a coupled fixed point $\left(x^{*}, y^{*}\right)$.

Now, we assume that $\left(x^{*}, y^{*}\right)$ and $\left(u^{*}, v^{*}\right)$ are two coupled fixed points of $F$ and that $x^{*}$ is comparable with $u^{*}$ and $y^{*}$ is comparable with $v^{*}$. If $u^{*} \succeq x^{*}$ and $y^{*} \succeq v^{*}$, using (8), we deduce that

$$
p\left(x^{*}, u^{*}\right)=p\left(F\left(x^{*}, y^{*}\right), F\left(u^{*}, v^{*}\right)\right) \leq \frac{p\left(x^{*}, x^{*}\right)+p\left(y^{*}, y^{*}\right)+p\left(u^{*}, u^{*}\right)+p\left(v^{*}, v^{*}\right)}{4} .
$$

Similarly, we deduce that

$$
p\left(y^{*}, v^{*}\right)=p\left(F\left(y^{*}, x^{*}\right), F\left(v^{*}, u^{*}\right)\right) \leq \frac{p\left(x^{*}, x^{*}\right)+p\left(y^{*}, y^{*}\right)+p\left(u^{*}, u^{*}\right)+p\left(v^{*}, v^{*}\right)}{4} .
$$

By summing up the two inequalities above, we get

$$
p\left(x^{*}, u^{*}\right)+p\left(y^{*}, v^{*}\right) \leq \frac{p\left(x^{*}, x^{*}\right)+p\left(y^{*}, y^{*}\right)+p\left(u^{*}, u^{*}\right)+p\left(v^{*}, v^{*}\right)}{2},
$$

that is, $p^{s}\left(x^{*}, u^{*}\right)+p^{s}\left(y^{*}, v^{*}\right)=0$ and hence $x^{*}=u^{*}$ and $y^{*}=v^{*}$. The same conclusion holds if $u^{*} \succeq x^{*}$ and $v^{*} \succeq y^{*}, x^{*} \succeq u^{*}$ and $y^{*} \succeq v^{*}$ or $x^{*} \succeq u^{*}$ and $v^{*} \succeq y^{*}$.

\section{Coupled Fixed Point under Suzuki-Type Condition}

Remark 13. Let $(X, d)$ be a metric space. Define $D: X^{2} \times X^{2} \rightarrow[0,+\infty)$ by

$$
D((x, y),(u, v))=d(x, u)+d(y, v), \quad \text { for all }(x, y),(u, v) \in X^{2} .
$$

It is simple to check that $D$ is a metric on $X^{2}$ and that $\left(X^{2}, D\right)$ is a complete metric space if $(X, d)$ is complete.

Remark 14. If $(X, \preceq)$ is a partially ordered set and if a relation $\sqsubseteq$ is defined on $X^{2}$ by:

$$
(x, y) \sqsubseteq(u, v) \Leftrightarrow x \preceq u \quad y \succeq v,
$$

then $\left(X^{2}, \sqsubseteq\right)$ is also partially ordered.

The next theorem is one of the main results in [19].

Theorem 15 (Paesano and Vetro $[19])$. Let $(X, \preceq)$ be a partially ordered set and suppose that there exists a metric $d$ on $X$ such that the metric space $(X, d)$ is complete. Let $T: X \rightarrow X$ be an increasing mapping with respect to $\preceq$. Define a non-increasing function $\theta$ from $[0,1)$ onto $(1 / 2,1]$ by

$$
\theta(r)= \begin{cases}1 & \text { if } 0 \leq r \leq(\sqrt{5}-1) / 2, \\ (1-r) r^{-2} & \text { if }(\sqrt{5}-1) / 2<r<2^{-1 / 2}, \\ (1+r)^{-1} & \text { if } 2^{-1 / 2} \leq r<1\end{cases}
$$

Assume that there exists $r \in[0,1)$ such that

$$
\theta(r) d(x, T x) \leq d(x, y) \quad \text { implies } \quad d(T x, T y) \leq r d(x, y),
$$

for all comparable $x, y \in X$. If the following conditions hold:

(i) there exists $x_{0} \in X$ such that $x_{0} \preceq T x_{0}$,

(ii) for an increasing sequence $\left\{x_{n}\right\} \subset X$ converging to $x \in X$ we have $x_{n} \prec x$ for all $n$, 
(iii) for two nondecreasing sequence $\left\{x_{n}\right\},\left\{y_{n}\right\} \subset X$ such that $x_{n} \preceq y_{n}, x_{n} \rightarrow x$ and $y_{n} \rightarrow y$ we have $x \preceq y$,

then $T$ has a fixed point in X. Moreover, the fixed point of $T$ is unique if:

(iv) for all $x, y \in X$ that are not comparable there exists $v \in X$ comparable with $x$ and $y$.

Theorem 16. Let $(X, \preceq)$ be a partially ordered set and suppose that there exists a metric $d$ on $X$ such that the metric space $(X, d)$ is complete. Let $F: X \times X \rightarrow X$ be a mixed monotone mapping. Define $\theta:[0,1) \rightarrow(1 / 2,1]$ as in Theorem 15 and assume that there exists $r \in[0,1)$ such that

$$
\theta(r)[d(x, F(x, y))+d(y, F(y, x))] \leq d(x, u)+d(y, v)
$$

implies

$$
d(F(x, y), F(u, v))+d(F(y, x), F(v, u))) \leq r[d(x, u)+d(y, v)]
$$

for all $x, y, u, v \in X$, with $x \succeq u, \quad y \preceq v$. If the following conditions hold:

(a) if a non-decreasing sequence $\left\{x_{n}\right\}$ converges to $x$, then $x_{n} \preceq x$, for all $n \geq 0$,

(b) if a non-increasing sequence $\left\{y_{n}\right\}$ converges to $y$, then $y \preceq y_{n}$, for all $n \geq 0$,

(c) for two monotone sequences $\left\{x_{n}\right\},\left\{y_{n}\right\} \subset X$ such that $x_{n} \preceq y_{n}, x_{n} \rightarrow x$ and $y_{n} \rightarrow y$ we have $x \preceq y$,

(d) there exist $x_{0}, y_{0} \in X$ such that $x_{0} \preceq F\left(x_{0}, y_{0}\right)$ and $y_{0} \succeq F\left(y_{0}, x_{0}\right)$,

then $F$ has a coupled fixed point in X. Moreover, the coupled fixed point of $F$ is unique if:

(e) for all $(x, y),(u, v) \in X^{2}$ that are not comparable with respect to $\sqsubseteq$, there exists $(z, w) \in X^{2} \sqsubseteq$ comparable with $(x, y)$ and $(u, v)$, where $\sqsubseteq$ is as in Remark 14 .

Proof. In the complete ordered metric space $(X, D, \sqsubseteq)$, where $D$ is the metric on $X^{2}$ defined in Remark 13 and $\sqsubseteq$ is the relation defined in Remark 14, at first, define $H(\cdot, \cdot): X^{2} \rightarrow X^{2}$ by

$$
H(x, y)=(F(x, y), F(y, x)) \text { for all }(x, y) \in X^{2} .
$$

If for some $(x, y),(u, v) \in X^{2}$, we have $\theta(r) D((x, y), H(x, y)) \leq D((x, y),(u, v))$, by Remark 13 , then

$$
\theta(r)[d(x, F(x, y))+d(y, F(y, x))] \leq[d(x, u)+d(y, v)] .
$$

This implies

$$
d(F(x, y), F(u, v))+d(F(y, x), F(v, u))) \leq r[d(x, u)+d(y, v)],
$$

and hence, by Remark 13, we get

$$
D(H(x, y), H(u, v)) \leq r D((x, y),(u, v)),
$$

that is, $H$ satisfies condition (11) of Theorem 15. Clearly, by condition $(d)$, there exists $\left(x_{0}, y_{0}\right) \in X^{2}$ such that $\left(x_{0}, y_{0}\right) \sqsubseteq H\left(x_{0}, y_{0}\right)$. If $\left(x_{n}, y_{n}\right)$ is a non-decreasing sequence in $X^{2}$ with respect to $\sqsubseteq$ such that $\left(x_{n}, y_{n}\right) \rightarrow(x, y)$, that is, such that $x_{n} \rightarrow x$ and $y_{n} \rightarrow y$. Then $\left\{x_{n}\right\}$ is a non-decreasing sequence with respect to $\preceq$ and $\left\{y_{n}\right\}$ a non-increasing sequence with respect to $\preceq$. Now, by $(a)$ and $(b)$, we have $x_{n} \preceq x$ and $y \preceq y_{n}$, and hence $\left(x_{n}, y_{n}\right) \sqsubseteq(x, y)$. Thus $(i i)$ of Theorem 15 holds. Similarly, by $(c)$, (iii) of Theorem 15 holds.

Let $(x, y) \sqsubseteq(u, v)$ then $x \preceq u$ and $v \preceq y$. Since $F$ has the mixed monotone property, then $F(x, y) \preceq$ $F(u, y)$ and $F(u, y) \preceq F(u, v)$ and so $F(x, y) \preceq F(u, v)$. Again, $F(v, u) \preceq F(v, x)$ and $F(v, x) \preceq F(y, x)$ and hence $F(v, u) \preceq F(y, x)$. This implies that $H(x, y) \sqsubseteq H(u, v)$. i.e., $\bar{H}$ is a non-decreasing mapping with respect to $\sqsubseteq$. Now, the hypotheses of Theorem 15 (that hold also if $T$ is non-decreasing) are satisfied and hence $H$ has a fixed point in $X^{2}$, say $\left(x^{*}, y^{*}\right)$. Then

$$
\left(F\left(x^{*}, y^{*}\right), F\left(y^{*}, x^{*}\right)\right)=H\left(x^{*}, y^{*}\right)=\left(x^{*}, y^{*}\right)
$$

i.e.,

$$
F\left(x^{*}, y^{*}\right)=x^{*} \text { and } F\left(y^{*}, x^{*}\right)=y^{*} .
$$

Now, if condition $(e)$ holds, then the coupled fixed point of $F$ is unique. 


\section{Conclusions}

Fixed point theory in partially ordered metric spaces is largely investigated due to its applications in pure and applied mathematics. Here, we established some results of existence and uniqueness of coupled fixed point, by using generalized contractive conditions in ordered partial metric spaces and ordered metric spaces. This kind of result is helpful to extend and complement existing theorems in the literature. An example illustrates the new theory.

\section{References}

1. R. P. Agarwal, M. A. El-Gebeily and D. O'Regan, "Generalized contractions in partially ordered metric spaces", Appl. Anal., vol. 87, 1-8, 2008.

2. I. Altun and H. Simsek, "Some fixed point theorems on ordered metric spaces and application", Fixed Point Theory Appl., vol. 2010, Article ID 621492, pp. 1-20, 2010.

3. A. Amini-Harandi and H. Emami, "A fixed point theorem for contraction type maps in partially ordered metric spaces and application to ordinary differential equations", Nonlinear Anal., vol. 72, pp. 2238-2242, 2010.

4. T. G. Bhaskar and V. Lakshmikantham, "Fixed point theorems in partially ordered metric spaces and applications", Nonlinear Anal., vol. 65, pp. 1379-1393, 2006.

5. B. Choudhury and A. Kundu, "A coupled coincidence point result in partially ordered metric spaces for compatible mappings", Nonlinear Anal., vol. 73, pp. 2524-2531, 2010.

6. Lj. B. Ćirić, N. Cakić, M. Rajović and J. S. Ume, "Monotone generalized nonlinear contractions in partially ordered metric spaces", Fixed point Theory Appl., vol. 2008, Article ID 131294, pp. 1-11, 2008.

7. Lj. B. Ćirić, B. Samet, H. Aydi and C. Vetro, "Common fixed points of generalized contractions on partial metric spaces and an application", Appl. Math. Comput., vol. 218, pp. 2398-2406, 2011.

8. J. Harjani and K. Sadarangani, "Generalized contractions in partially ordered metric spaces and applications to ordinary differential equations", Nonlinear Anal., vol. 72, pp. 1188-1197, 2010.

9. N. Hussain, M. H. Shah and M. A. Kutbi, "Coupled coincidence point theorems for nonlinear contractions in partially ordered quasi-metric spaces with a Q-function", Fixed point Theory Appl., vol. 2011, Article ID 703938, pp. 1-21, 2011.

10. V. Lakshmikantham and $\mathrm{Lj}$. B. Ćirić, "Coupled fixed point theorems for nonlinear contractions in partially ordered metric spaces", Nonlinear Anal., vol. 70, pp. 4341-4349, 2009.

11. S. G. Matthews, "Partial metric topology", in Proc. 8th Summer Conference on General Topology and Applications, Ann. New York Acad. Sci., vol. 728, 1994, pp. 183-197.

12. H. K. Nashine and I. Altun, "Fixed point theorems for generalized weakly contractive condition in ordered metric spaces", Fixed point Theory Appl., vol. 2011, Article ID 132367, pp. 1-20, 2011.

13. H. K. Nashine and B. Samet, "Fixed point results for mappings satisfying $(\psi, \varphi)$-weakly contractive condition in partially ordered metric spaces", Nonlinear Anal., vol. 74, pp. 2201-2209, 2011.

14. H. K. Nashine, B. Samet and C. Vetro, "Monotone generalized nonlinear contractions and fixed point theorems in ordered metric spaces", Math. Comput. Modelling, vol. 54, pp. 712-720, 2011.

15. S. J. O'Neill, "Partial metrics, valuations and domain theory", in Proc. 11th Summer Conference on General Topology and Applications, Ann. New York Acad. Sci., vol. 806, 1996, pp. 304-315.

16. J. J. Nieto and R. Rodríguez-López, "Contractive mapping theorems in partially ordered sets and applications to ordinary differential equations", Order, vol. 22, pp. 223-239, 2005.

17. J. J. Nieto and R. Rodríguez-López, "Existence and uniqueness of fixed point in partially ordered sets and applications to ordinary differential equations", Acta Math. Sinica (Engl. Ser.), vol. 23, pp 2205-2212, 2007.

18. S. Oltra and O. Valero, "Banach's fixed point theorem for partial metric spaces", Rend. Istit. Mat. Univ. Trieste, vol. 36, pp. 17-26, 2004.

19. D. Paesano and P. Vetro, "Suzuki's type characterizations of completeness for partial metric spaces and fixed points for partially ordered metric spaces", Topology Appl., vol. 159, pp. 911-920, 2012.

20. A. C. M. Ran and M. C. B. Reurings, "A fixed point theorem in partially ordered sets and some applications to matrix equations", Proc. Amer. Math. Soc., vol. 132, pp. 1435-1443, 2004.

21. D. O'Regan and A. Petrutel, "Fixed point theorems for generalized contractions in ordered metric spaces", $J$. Math. Anal. Appl., vol. 341, pp. 1241-1252, 2008.

22. S. Romaguera, "A Kirk type characterization of completeness for partial metric spaces", Fixed Point Theory Appl., vol. 2010, Article ID 493298, pp. 1-6, 2010.

23. B. Samet, "Coupled fixed point theorems for a generalized Meir-Keeler contraction in partially ordered metric spaces", Nonlinear Anal., vol. 72, pp. 4508-4517, 2010. 
24. W. Shatanawi, "Partially ordered cone metric spaces and coupled fixed point results", Comput. Math. Appl., vol. 60, pp. 2508-2515, 2010.

25. O. Valero, "On Banach fixed point theorems for partial metric spaces", Appl. Gen. Topol., vol. 6, pp. 229-240, 2005.

26. Y. Wu, "New fixed point theorems and applications of mixed monotone operator", J. Math. Anal. Appl., vol. 341, pp. 883-893, 2008.

27. Y. Wu and Z. Liang, "Existence and uniqueness of fixed points for mixed monotone operators with applications", Nonlinear Anal., vol. 65, pp. 1913-1924, 2006. 